\title{
Detection of vertical slope movement in highly vegetated tropical area of Gunung pass landslide, Malaysia, using L-band InSAR technique
}

\begin{abstract}
Landslides are one of the most dangerous natural hazards in the world which have significant negative impact on so many lives and properties. Interferometric Synthetic Aperture Radar (InSAR) is an imaging technique for measuring changes in the detailed characteristics of the surface which is timely and cost effective. This research aimed to detect the landslide that occurred in Gunung pass area, Malaysia using InSAR generated from ALOSPALSAR repeat pass data. The signals information was converted into amplitude and phase for both scenes where the phases were used to construct the InSAR. Goldstein filter was used to reduce the phase noise and the results were used as an input for phase unwrapping. Using the unwrapped phase, the vertical displacement was measured and landslide was recognized. Results showed the efficiency of InSAR in detecting the movement of landslide in Gunung pass without the differential having to generate DInSAR. The results were validated using the observed reference point of the landslides and the root mean square error (RMSE) was 0.19. Furthermore, advance 3D processing was performed for measuring the volume of the landslides. The achievements of current research represented that PALSAR data yield excellent performance to generate the interferometric and landslide could be detected very precisely in highly vegetated tropical forest.
\end{abstract}

Keyword: Synthetic Aperture Radar (SAR); Landslide; InSAR; Gunung pass; Remote sensing; GIS; ALOS PALSAR 